\title{
Amplitude Instability of Somatosensory Evoked Potentials as an Indicator of Delayed Cerebral Ischemia in a Case of Subarachnoid Hemorrhage
}

Clinical EEG and Neuroscience 2019, Vol. 50(3) 205-209 (C) EEG and Clinical Neuroscience Society (ECNS) 2018

Article reuse guidelines: sagepub.com/journals-permissions DOI: 10.1 I77//5500594/88049/5 journals.sagepub.com/home/eeg (S)AGE

\author{
Maddalena Spalletti' ${ }^{1}$, Vanni Orzalesi ${ }^{2}$, Riccardo Carrai ${ }^{1,3}$, \\ Luca Bucciardini', Cesarina Cossu', Maenia Scarpino', ${ }^{1,3}$, Enrico Fainardi ${ }^{4,5}$, \\ Marinella Marinoni ${ }^{1,6}$, Antonello Grippo ${ }^{1,3}$, and Aldo Amantini ${ }^{1,3}$
}

\begin{abstract}
We describe a 55-year-old male patient with a subarachnoid hemorrhage (SAH) as a result of left middle cerebral artery (MCA) aneurysm rupture, who underwent continuous electroencephalogram (EEG) and somatosensory evoked potential (cEEG-SEP) monitoring that showed an unusual SEP trend pattern. EEG was continuously recorded, and SEPs following stimulation of median nerves were recorded every 50 minutes, with the amplitude and latency of the cortical components automatically trended. An increase in intracranial pressure required a left decompressive craniectomy. cEEG-SEP monitoring was started on day 7, which showed a prolonged (24 hours) instability of SEPs in the left hemisphere. During this phase, left MCA vasospasm was demonstrated by transcranial Doppler (TCD), and computed tomography perfusion (CTP) showed a temporo-parietooccipital ischemic penumbra. Following intravascular treatment, hypoperfusion and the amplitude of cortical SEPs improved. In our case, a prolonged phase of SEP amplitude instability during vasospasm in SAH correlated with a phase of ischemic penumbra, as demonstrated by CTP. In SAH, SEP instability during continuous monitoring is a pattern of alert that can allow treatments capable of avoiding irreversible neurological deterioration.
\end{abstract}

\section{Keywords}

continuous SEP monitoring, neurophysiological monitoring, subarachnoid hemorrhage, acute brain injury, delayed cerebral ischemia

Received July 13, 2018; revised September II, 2018; accepted September 12, 2018.

\section{Introduction}

Subarachnoid hemorrhage (SAH) is frequently associated with vasospasm and delayed cerebral ischemia (DCI), which are major causes of mortality and morbidity. ${ }^{1-3}$ The pathogenesis of DCI is complex and only partially understood; it is not limited to large vessel vasospasm, but is based on various hemodynamic disturbances, including microvascular dysfunction, which finally lead to ischemia. ${ }^{1,3,4}$ An optimal DCI monitoring strategy has still not been established for comatose, clinically nonevaluable patients. At present, the monitoring strategy is based on a multimodal approach, with most methods aimed at detecting hemodynamic alterations, such as digital subtraction angiography (DSA), angio-computed tomography (CT) or angio-magnetic resonance imaging (MRI), CT-perfusion (CTP) or MRI-perfusion, transcranial Doppler (TCD), and invasive monitoring of intracranial pressure. Neuronal function, however, cannot be directly assessed by these methods, but only by neurophysiological tools, in particular electroencephalogram (EEG) and somatosensory evoked potentials (SEPs), which are highly sensitive to ischemia. ${ }^{5}$ Some authors have proposed quantitative-EEG (QEEG) for the detection of DCI, ${ }^{2,6,7}$ but agreement regarding which parameters should be considered

\footnotetext{
'SOD Neurofisiopatologia, Dipartimento Neuromuscoloscheletrico e Organi di Senso, AOU Careggi, Florence, Italy

${ }^{2}$ SOD Neuroanestesia e Rianimazione, Dipartimento di Anestesia e Rianimazione, AOU Careggi, Florence, Italy

${ }^{3}$ IRCCS, Fondazione Don Carlo Gnocchi, Florence, Italy

${ }^{4}$ SOD Neuroradiologia, AOU Careggi, Florence, Italy

5Dipartimento di Scienze Biomediche Cliniche e Sperimentali "Mario Serio", Università degli Studi di Firenze, Florence, Italy

${ }^{6}$ Unit Neurosonologia, Dipartimento Neuromuscoloscheletrico e Organi di Senso, AOU Careggi, Florence, Italy

Corresponding Author:

Maddalena Spalletti, SOD Neurofisiopatologia, Dipartimento

Neuromuscoloscheletrico e Organi di Senso, AOU Careggi, Largo Brambilla 3, Florence 50134, Italy.

Email: maddalena@spalletti.it
}

Full-color figures are available online at journals.sagepub.com/home/eeg 


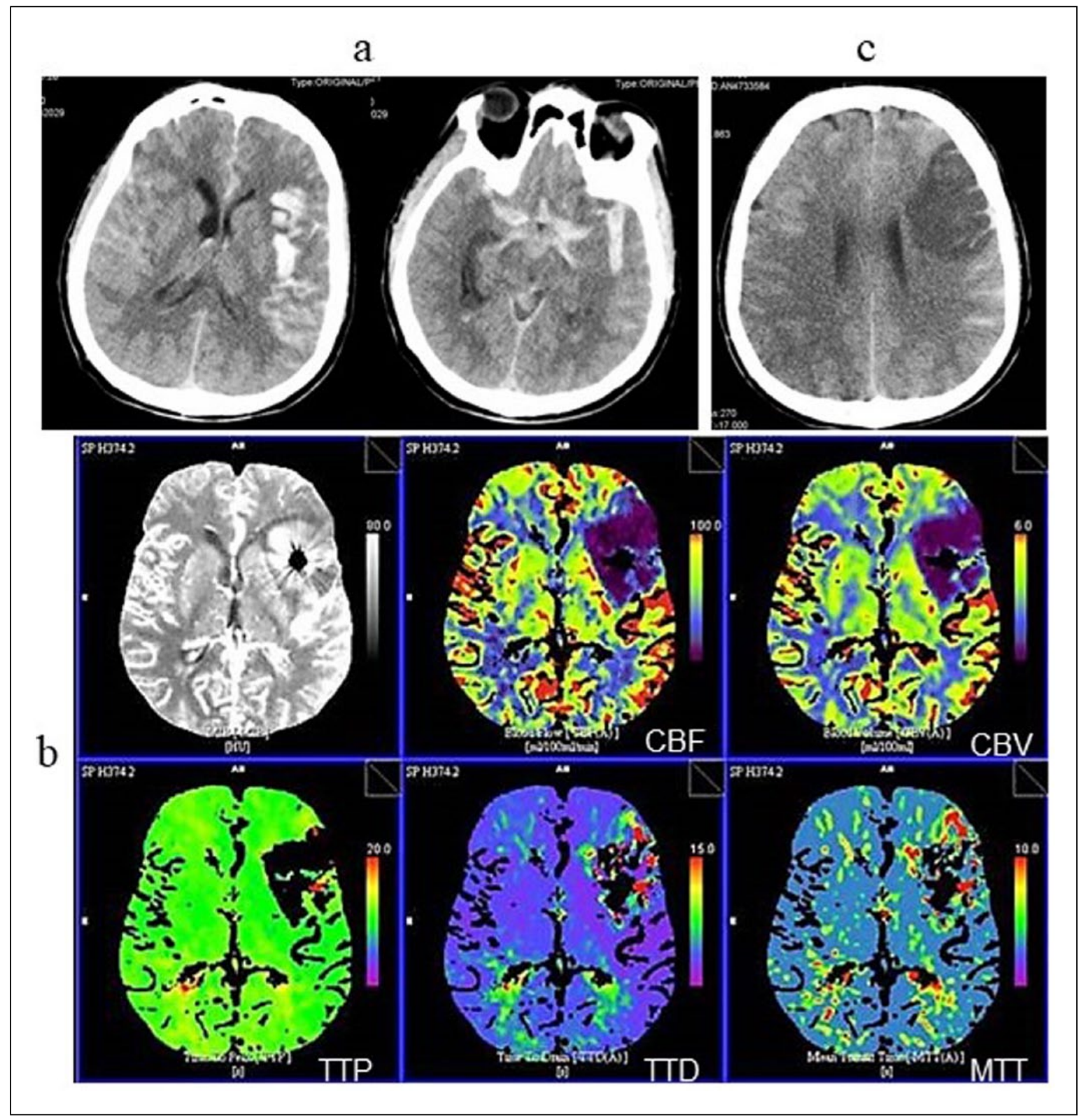

Figure I. (a) Computed tomography (CT) scan prior to angiographic treatment: large subarachnoid hemorrhage with prevalence in the basal cisternae and in the left Sylvian region. (b) CT perfusion [CTP] the day after angiographic treatment (average CTP maps, from top left to bottom right: cerebral blood flow [CBF], cerebral blood volume [CBV], time to peak [TTP], time to drain [TTD], and mean transit time [MTT]). Left fronto-insular area showing very low CBF and CBV and high TTD and MTT values suggestive of an infarct core without ischemic penumbra, corresponding to a hypodense ischemic area on the CT scan. No further areas with abnormal perfusion values were identified. (c) CT scan the day after angiographic treatment showing the left fronto-insular ischemic area.

has not been reached $;{ }^{8}$ moreover, neurosedation can alter most of QEEG parameters.

SEPs are resistant to sedatives and easily transformed into amplitude and latency trends; nonetheless, reports of continuous SEP or EEG-SEP monitoring are scarce. -11 $^{-11}$

Here, we monitored a case of SAH by means of continuous EEG-SEPs (cEEG-SEPs), in which SEPs showed ischemic changes, while the EEG was relatively uninformative due to neurosedation.

\section{Case Report}

A 55-year-old male patient was found comatose with a Glasgow Coma Scale (GCS) of 3. Brain CT and angio-CT scans revealed a massive subarachnoid hemorrhage (Fisher scale $=4$, Figure 1a) due to a dissecting aneurysm of the frontal branch of the left middle cerebral artery (MCA) with distal occlusion. Endovascular treatment was successfully performed by parent vessel occlusion, and the patient was transferred to the 
neurological intensive care unit (NICU), where he was moderately sedated. Clinically, the patient was right hemiplegic, with $\mathrm{GCS}=6$ (motor score $=4$ in the left limbs). The following day, CTP (Figure 1b) showed a left fronto-temporo-insular ischemic area characterized by low cerebral blood flow $(\mathrm{CBF})$ and cerebral blood volume (CBV) and high mean transit time (MTT), time to peak (TPP), and time to drain (TTD) values, which were indicative of an infarct core corresponding to the avascular area previously found at DSA and to the hypodense lesion detected on the CT scan acquired immediately prior to CTP (Figure 1c).

From day 4, the intracranial pressure (ICP) increased (with peaks up to $40 \mathrm{~mm} \mathrm{Hg}$ ): at first it responded to medical management, but then the increase became refractory to therapies; therefore, a left decompressive craniectomy was performed on day 6 .

On day 7, the ICP was still increased $(23-28 \mathrm{~mm} \mathrm{Hg})$, and TCD showed a mild left MCA vasospasm $(130 \mathrm{~cm} / \mathrm{s}$, MCA/ internal carotid artery $[\mathrm{ICA}]$ ratio $=3$ ). CEEG-SEP monitoring (Nemus-ICU, EBNeuro, Florence, Italy) was applied as described previously. ${ }^{12}$ In brief, stainless steel needle electrodes were used for recording both EEG (F3-C3, C3-T3, T3-P3; F4-C4, C4-T4, T4-P4) and SEPs (ipsilateral referred to contralateral Erb's point, $\mathrm{C}^{\prime} / \mathrm{C}^{\prime}$ ' referred to $\mathrm{Fz}$ ) and for alternately stimulating the right and left median nerve at the wrist (right/left interstimulus interval $333 \mathrm{~ms}$, pulse duration $0.2 \mathrm{~ms}$, stimulus rate $3 \mathrm{~Hz}$, stimulus intensity 5-10 $\mathrm{mA}$ above the motor threshold). EEG was continuously recorded; SEPs were acquired in 12-minute sessions every 50 minutes; raw EEG, EEG density spectral array (computed for C3-P3 and C4-P4), SEPs (viewed in cascades), and SEP trends (absolute amplitude and latency plotted against time) were displayed on the screen. The ongoing recording was constantly available to the NICU staff at the bedside and remotely in the Neurophysiology Unit to the neurophysiologists (during daytime from Monday to Saturday).

At the beginning of the monitoring (day 7), SEPs were bilaterally present and normal in amplitude, and the EEG showed a burst-suppression pattern due to sedation (propofol $3 \mathrm{mg} / \mathrm{kg} / \mathrm{h}$, midazolam $0.5 \mathrm{mg} / \mathrm{kg} / \mathrm{h}$, fentanyl $4 \mu \mathrm{g} / \mathrm{kg} / \mathrm{h}$ ). During days 7 and 8 , the ICP was controlled and stabilized at values $<20 \mathrm{~mm}$ $\mathrm{Hg}$. On the following night, left cortical SEPs showed a persistent $40 \%$ decrease in amplitude with regard to the template. A brain CT performed after 24 hours revealed a new small left subcortical parietal ischemic area.

On day 10, despite the ICP being only slightly altered (19-24 $\mathrm{mm} \mathrm{Hg}$ ), SEPs in the left hemisphere showed a phase of high instability lasting approximately 24 hours (repeated amplitude reductions ranging from $40 \%-50 \%$ to $80 \%$, followed by partial amplitude recoveries) that was unrelated to variations in the ICP (Figure 2a). The EEG showed a slow diffuse low-voltage discontinuous pattern without clear asymmetry on EEG. On day 11, TCD showed further acceleration of the left MCA (160 $\mathrm{cm} / \mathrm{s}, \mathrm{MCA} / \mathrm{ICA}$ ratio $=4.5)$, the ICP was unchanged $(20-25$ $\mathrm{mm} \mathrm{Hg}$ ), angio-CT demonstrated a left MCA vasospasm, and CTP showed the occurrence of a corresponding new left temporo-parieto-occipital area of hypoperfusion close to the already-known infarct core, characterized by a pattern indicative of ischemic penumbra (Figure 2b: low values of CBF, high values of MTT, TPP, TTD, normal values of CBV). DSA confirmed severe vasospasm and an avascular profile in the same areas. Treatment with locoregional infusion of nimodipine in conjunction with angioplasty improved the MCA caliber.

During this phase, the EEG-SEP monitoring was interrupted to allow frequent transfer of the patient. Despite the severity of the neuroradiological and TCD findings, the ICP was sufficiently controlled with mild sedation, and CTP on day 12 showed a clear improvement of hypoperfusion in the temporoparieto-occipital areas. On the same day, cEEG-SEP monitoring was restored, and left cortical SEP progressively improved, with the final left SEP amplitude being restored to values preceding the phase of SEP instability. Serial TCD showed resolution of the vasospasm from day 13, and the neurological conditions further improved (GCS: eye score $=4$, verbal score $=1$, motor score $=5$ ); cEEG-SEP monitoring was definitively interrupted on day 18 .

After 50 days, the patient was aphasic, right hemiparetic but conscious, with no further ischemic areas visible on the brain CT scan, in particular in the left temporo-parieto-occipital areas.

The patient's caregiver provided informed consent for publication.

\section{Discussion}

In our patient, we observed 2 types of SEP changes that correlated well with different CT and CTP findings (ischemic core and ischemic penumbra). We first observed a stable $40 \%$ amplitude reduction in the left cortical SEP amplitude (day 9) related to a new small left parietal ischemic area on CT, in the context of a mild MCA vasospasm as seen on TCD. On day 10, the left cortical SEP showed prolonged amplitude instability lasting for 24 hours; since the CTP showed left parietal ischemic penumbra on day 11 , we interpreted the SEP instability in the left hemisphere as a variable hypoperfusion fluctuating between oligemia and ischemic penumbra, approaching the thresholds of synaptic failure. ${ }^{5}$

This hypoperfusion was documented on CTP, with values compatible with ischemic penumbra in the left temporo-parieto-occipital areas. Subsequently, no further areas of ischemic cores were identified, and this was confirmed by the overall response to treatment: improvement in TCD, CTP, and angiographic findings, no new ischemic areas on $\mathrm{CT}$, and remission of left cortical SEP instability with the final SEP amplitude being restored to pre-instability levels.

In previous studies, we have reported trend instability at cEEG-SEP monitoring as a pattern preceding the disappearance of cortical SEPs related to irreversible deterioration of brain function. ${ }^{9}$ In the present case, a stable reduction in left cortical SEP amplitude corresponded to the new onset of a small irreversible ischemic core, but most notably, in the following days we then observed an instability in the SEP trend in the same hemisphere, which, also evaluating the TCD and neuroimaging findings, we attributed to reversible ischemia.

This SEP pattern solicited prompt treatment that likely prevented the progression of a reversible ischemic process toward irreversible neuronal damage. 


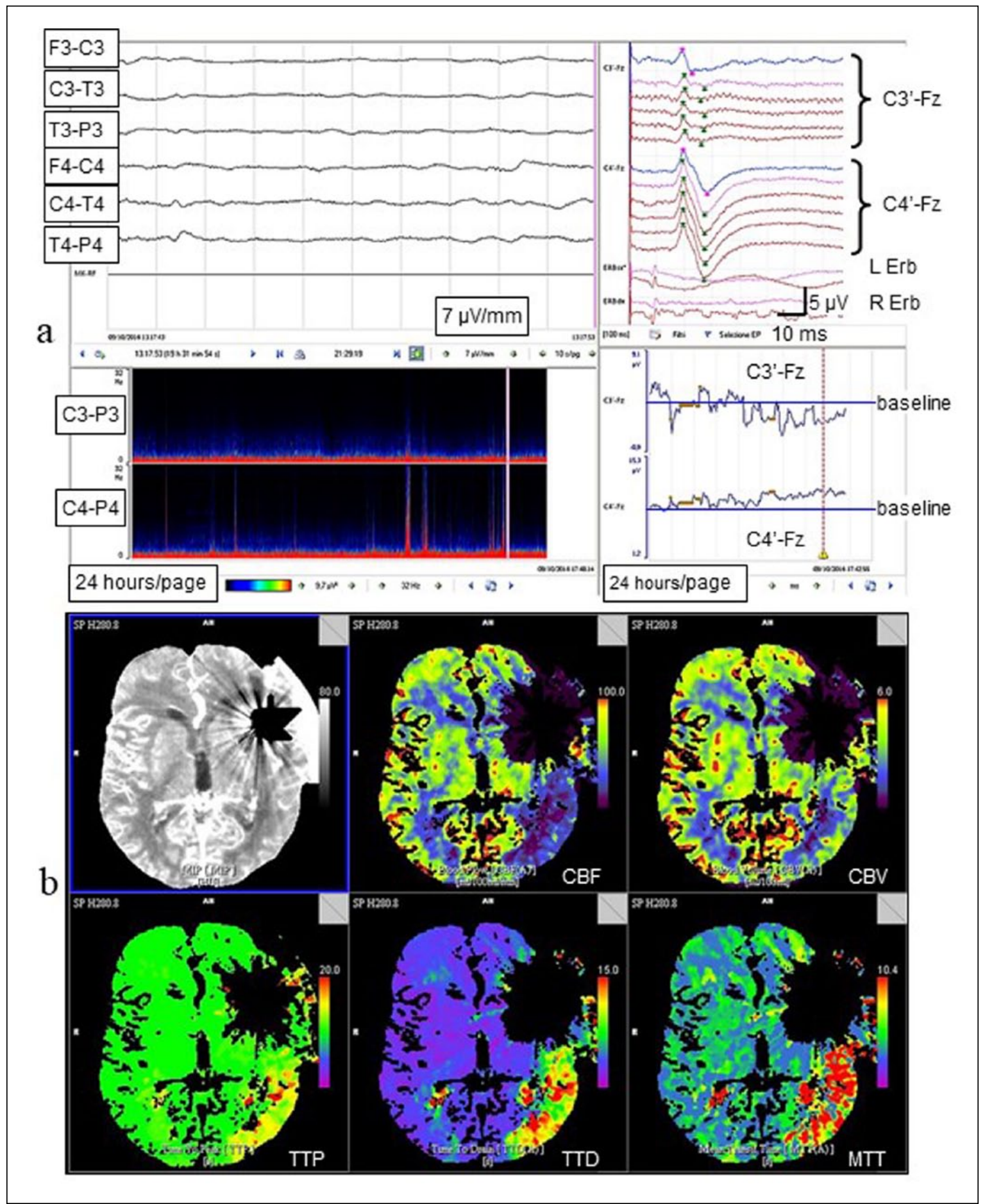

Figure 2. Continuous electroencephalogram-somatosensory evoked potential (cEEG-SEP) monitoring. (a) SEP panel on the right showing changes in left cortical SEP amplitude: SEP cascades (top) show reduced amplitude in the left hemisphere with regard to the blue template; amplitude trends (bottom) show instability of the left cortical SEP (horizontal blue lines represent baselines, i.e., amplitude of the template for each hemisphere). EEG panel on the left: raw EEG (top) with a discontinuous low-voltage pattern, density spectral array (bottom) with no significant variations in either hemisphere. (b) Computed tomography perfusion [CTP] (average CTP maps, from top left to bottom right: cerebral blood flow [CBF], cerebral blood volume [CBV], time to peak [TTP], time to drain [TTD], and mean transit time [MTT]) showing a large left fronto-temporo-parietal area of hypoperfusion with altered values in the CBF, TPP, TTD, and MTT maps, while CBV is normal; this pattern is suggestive of ischemic penumbra. 
While cEEG in brain-injured patients has become common in most NICUs, to the best of our knowledge, only a few previous studies ${ }^{9-11}$ have applied cEEG-SEP monitoring. In our patient, SEP monitoring was more informative than EEG, allowing reliable evaluation even with high levels of sedation, during which EEG and clinical examination were relatively uninformative. In fact, EEG showed a burst-suppression or slow diffuse low-voltage pattern, making the quantitative parameters proposed by others ${ }^{2,6,7}$ (alpha/delta ratio, alpha variability) scarcely assessable. We are aware that SEPs explore a relatively small cortical (parietal) area as compared with EEG, and this represents a limitation of their use; however, the parietal cortex belongs to the distribution territory of the MCA, which represents approximately $70 \%$ of hemispheric perfusion.

In our patient, an uncommon unilateral instability of SEP amplitude at cEEG-SEP monitoring, further supported by findings of worsened ipsilateral MCA vasospasm on TCD, warned the NICU staff to proceed promptly to a more thorough vasospasm assessment. Therefore, the question arises whether cEEG-SEP monitoring could be included in a generally accepted multimodal stepwise assessment of vasospasm from TCD to DSA. ${ }^{13}$ If our findings are confirmed in larger studies, we think that cEEG-SEP monitoring could make a valuable contribution to the decision whether to progress to non-bedside techniques such as angio-CT, CTP, and/or DSA.

\section{Author Contributions}

Maddalena Spalletti contributed to conception and design of the study and contributed to data acquisition, analysis, and interpretation; drafted and critically revised the manuscript; gave final approval; agrees to be accountable for all aspects of work ensuring integrity and accuracy. Vanni Orzalesi contributed to conception of the study and to data acquisition and interpretation; critically revised the manuscript; gave final approval; agrees to be accountable for all aspects of work ensuring integrity and accuracy.Riccardo Carrai contributed to conception of the study and to data analysis and interpretation; critically revised the manuscript; gave final approval; agrees to be accountable for all aspects of work ensuring integrity and accuracy. Luca Bucciardini conributed to conception of the study and to data acquisition and interpretation; critically revised the manuscript; gave final approval; agrees to be accountable for all aspects of work ensuring integrity and accuracy. Cesarina Cossu contributed to data acquisition; critically revised the manuscript; gave final approval; agrees to be accountable for all aspects of work ensuring integrity and accuracy. Maenia Scarpino contributed to conception of the study; critically revised the manuscript; gave final approval; agrees to be accountable for all aspects of work ensuring integrity and accuracy. Enrico Fainardi contributed to data analysis and interpretation; critically revised the manuscript; gave final approval; agrees to be accountable for all aspects of work ensuring integrity and accuracy. Marinella Marinoni contributed to data acquisition; critically revised the manuscript; gave final approval; agrees to be accountable for all aspects of work ensuring integrity and accuracy. Antonello Grippo contributed to conception and design of the study and to data analysis and interpretation; critically revised the manuscript; gave final approval; agrees to be accountable for all aspects of work ensuring integrity and accuracy. Aldo Amantini contributed to conception and design of the study and to data analysis and interpretation; drafted and critically revised the manuscript; gave final approval; agrees to be accountable for all aspects of work ensuring integrity and accuracy.

\section{Declaration of Conflicting Interests}

The author(s) declared no potential conflicts of interest with respect to the research, authorship, and/or publication of this article.

\section{Funding}

The author(s) received no financial support for the research, authorship, and/or publication of this article.

\section{ORCID iD}

Maddalena Spalletti (iD https://orcid.org/0000-0002-6526-0722

\section{References}

1. Foreman B. The pathophysiology of delayed cerebral ischemia. J Clin Neurophysiol. 2016;33:174-182. doi:10.1097/ WNP.0000000000000273

2. Rots ML, van Putten MJ, Hoedemaekers CW, Horn J. Continuous EEG monitoring for early detection of delayed cerebral ischemia in subarachnoid hemorrhage: a pilot study. Neurocrit Care. 2016;24:207-216.

3. Francoeur CL, Mayer SA. Management of delayed cerebral ischemia after subarachnoid hemorrhage. Crit Care. 2016;20:277.

4. Mir DI, Gupta A, Dunning A, et al. CT perfusion for detection of delayed cerebral ischemia in aneurysmal subarachnoid hemorrhage: a systematic review and meta-analysis. AJNR Am J Neuroradiol. 2014;35:866-871.

5. Astrup J, Siesjo BK, Symon L. Thresholds in cerebral ischemiathe ischemic penumbra. Stroke. 1981;12:723-725.

6. Claassen J, Velazquez A, Meyers E, et al. Bedside quantitative electroencephalography improves assessment of consciousness in comatose subarachnoid hemorrhage patients. Ann Neurol. 2016;80:541-553.

7. Vespa PM, Nuwer MR, Juhász C, et al. Early detection of vasospasm after acute subarachnoid hemorrhage using continuous EEG ICU monitoring. Electroencephalogr Clin Neurophysiol. 1997;103:607-615.

8. Kondziella D, Friberg CK, Wellwood I, Reiffurth C, Fabricius M, Dreier JP. Continuous EEG monitoring in aneurysmal subarachnoid hemorrhage: a systematic review. Neurocrit Care. 2015;22:450-461.

9. Amantini A, Fossi S, Grippo A, et al. Continuous EEG-SEP monitoring in severe brain injury. Neurophysiol Clin. 2009;39: 85-93.

10. Bosco E, Marton E, Feletti A, et al. Dynamic monitors of brain function : a new target in neurointensive care unit. Crit Care. 2011;15:R170.

11. Moulton R, Kresta P, Ramirez M, Tucker W. Continuous automated monitoring of somatosensory evoked potentials inposttraumatic coma. J Trauma. 1991;31:676-683.

12. Fossi S, Amantini A, Grippo A, et al. Continuous EEG-SEP monitoring of severely brain injured patients in NICU: methods and feasibility. Neurophysiol Clin. 2006;36:195-205.

13. Bouzat P, Payen J, Crippa IA, Taccone FS. Noninvasive vascular methods for detection of delayed cerebral ischemia after subarachnoid hemorrhage. J Clin Neurophysiol. 2016;33:260-267. 